\title{
Seismic retrofitting on structures in urban areas
}

\author{
A. K. Desai \\ Applied Mechanics Department, S V National Institute of Technology, \\ Surat, India
}

\begin{abstract}
After the Bhuj earthquake event in 2001, earthquakes and seismic hazards are increasing as the Indian continent (Tectonic Plate) moves slowly towards the North-East Himalayan Mountain side. Now, because of the collision of two tectonic plates and due to a folding of the plate, the top crust is subjected to tension cracking. This is the main cause of earthquakes in the Asian region. The frequency of earthquake shocks from different epicenters is increasing as compared to previous shocks.

The Indian government modified its seismic design code from I.S.: 18931984 to I.S.: 1893-2002, for seismic safety in its newly designed buildings. However, problems do arise for old structures, as quite a large part of the Indian population resides in urban areas and some even in poorly constructed RCC buildings, with a seismic risk zone from Zone-III to Zone-IV (most dangerous). The only option remaining for local authorities for reduction of earthquake hazards and for increasing the safety of the urban population is for "Seismic Retrofitting" of old structures. Three aspects are most important for prioritization of structures that need retrofitting

1. Structures of national importance, lifeline and critical facilities.

2. Multi-storied residential and commercial complex.

3. Only ground floor/ low rise residential building.

Again, structural auditing for three aspects are required for priority of retrofitting structures near to collapse, but can be refortified and require steel probe supporting; main structural components like beams and columns that require immediate retrofitting at some places; structures can be made safe following this old design code, but retrofitting is required from a new design code for higher level earthquakes. After mixing the above two auditing criteria, the availability of a
\end{abstract}


"Retrofitting fund" and cost effective "Retrofitting method" must be thought of, for urban life safety.

Keywords: effect of earthquake, retrofitting method, old and new building audit, seismic hazard, safety.

\section{Introduction}

Earthquakes can pose one of the greatest challenges to the designer of buildings and other civil engineering structures. The potential for violent ground motions lasting not more than a few minutes to cause great destruction has been amply demonstrated by recent events.

As narrated by Shah et al. [1], lessons learnt from the Bhuj earthquake (26/01/2001), in combination with research efforts and new technologies, lead to changes in the I.S. Code (1893-2001) of practice, for designing more reliable structures. But what to do with those existing structures which were constructed before the imposing of advance seismic code or structures that were built with inherent conceptual detailing or construction error.

Experience from past earthquakes and results of structural analysis indicate that a large proportion of the existing reinforced concrete buildings in Gujarat (or any) is vulnerable to damage or even collapse during a strong earthquake. These structures neither possess sufficient strength nor ductility to perform satisfactorily during future earthquakes. Consequently, there is a need to redesign all structures constructed in the past, and not just which have been damaged during earthquakes. However, the cost of redesigning all damaged structures will be too high and also "old" structures which provide essential service such as hospitals, schools and telecommunication structure should be protected, not only to avoid collapse, but also to ensure that important social function are maintained even after a strong seismic shock. So retrofitting of certain structures is essential.

\subsection{Characteristics of earthquake effects on structures}

Some of the key factors which differentiate the effects of earthquakes' strong motion from other types of loading are listed below.

i Earthquake loading arises from ground accelerations causing inertial forces within a structure. These are dynamic effects i.e. the dynamic properties of the structure determine the severity of the response.

ii Earthquake loading is cyclic in nature, and the potentially degrading effect of such loading on structures and foundation soils must be taken into consideration

iii There is great uncertainty in the amplitude, duration and frequency content of the motions that may be expected at a particular site, moreover, the response of structures to such complex motions, even if these motions were known with certainty, is often difficult to predict with confidence.

iv Earthquake motions with a long return period (very low annual probability) are proportionately large compared with short-return period motions. 
There are approximately 120 million buildings in seismic Zones III, IV and V. Most of these buildings are not earthquake-resistant and are potentially vulnerable to collapse in the event of a high intensity earthquake. It is not practically feasible or financially viable to retrofit all the existing buildings. The guidelines given by the National Disaster Management Authority (Government of India) are helpful in selecting the buildings which need seismic strengthening. These guidelines recommend the structural safety audit and retrofitting of select critical lifeline structures and high priority buildings.

\subsection{Prioritization of structures}

The initial focus for structural safety audit and retrofitting will be on government and public buildings. For private buildings necessary capacity for carrying out assessments is to be developed through suitable capacity development efforts among the professionals in the private sector.

Here is an illustrative priority list for Structural Safety Audit, Seismic 1.3 Strengthening of structures.

Buildings of national importance like Rashtrapati Bhavan, Parliament House, the Supreme Court of India, Raj Bhavans, Legislatures, High Courts, Central and State Secretariats, historical monuments, museums, heritage buildings, strategic assets and vital installations such as power plants.

Lifeline buildings, structures and critical facilities like schools, colleges and academic institutions; hospitals and health facilities, tertiary care centers and all hospitals designated as major hospitals.

i Public utility structures like reservoirs and dams; bridges and flyovers; ports and harbors; airports, railway stations and bus station complexes.

ii Important buildings that ensure governance and business continuity like offices of the district collector and superintendent of police in districts; buildings of financial institutions like the Reserve Bank of India and the stock exchanges.

iii Multi-storied buildings with five or more floors in residential apartments, office and commercial complexes.

However the responsibility to identify and prioritize these structures will rest with respective state governments.

\subsection{Structural safety audit of critical lifeline structures}

The seismic risk profile can be quantified only after the vulnerability of building inventory in a geographic area is compiled. Assessment techniques can be used to determine the vulnerability of the buildings, in order of priority. Two levels of seismic vulnerability assessment can be carried out for buildings, namely Rapid Visual Screening (RVS) and Detailed Vulnerability Assessment (DVA). The former is a quick visual estimation but cannot give detailed technical information of structures to determine whether the structure is considered to be vulnerable or not. Once the RVS identifies a structure to be vulnerable, then that structure is subjected to a detailed assessment for a quantitative evaluation of its 
vulnerability. For structures other than buildings, DVAs are normally carried out. A DVA consists of evaluating the structural systems that resist the earthquake loads, as well as assessing non-structural elements like the contents, finishes and elements that do not resist any earthquake load of the structure.

RVS procedures need to be developed for all types of building systems in India.

\subsection{Seismic strengthening and retrofitting}

The seismic strengthening and retrofitting of some fragile lifeline structures is undertaken through a pilot project being implement by Government of India. The prioritization of the cities is based on the

- degree of seismic hazard,

- population size,

- level of vulnerability of the building/structure,

- importance of the structure, and

- the speed with which the states can undertake these initiatives.

Accomplishing seismic retrofitting of the existing built environment requires a systematic and sustained effort, by carrying out several activities in each of the towns and cities. These activities are:

- Developing an inventory of the existing built environment.

- Assessing the vulnerability of these constructions.

- Prioritizing structures found vulnerable.

- Developing seismic retrofitting measures.

- Undertaking construction work to strengthen vulnerable structures.

\section{Cracks and earthquake affected structures}

As per I.S. 13935:1993 [2] for repair and seismic strengthening of building guidelines it is classified as per width of cracks

i Minor cracks - These cracks are very fine cracks. Generally shown in concrete structure, only a crack width near a $0.5 \mathrm{~mm}$ is called minor cracks. These are also called a hair cracks.

ii Medium cracks - The crack width near $0.5 \mathrm{~mm}$ to $5 \mathrm{~mm}$ called as a medium cracks. Generally, observed in masonry and concrete.

iii Major cracks - The crack width wider than about $5 \mathrm{~mm}$ is called major cracks. Generally it is shown in masonry and concrete.

As presented by Jain Sudhir and Murty [3] crack patterns are very important in seismic engineering, because by using cracks patterns we can judge the effect of earthquakes on a building. For the repair of minor and medium cracks $(0.50 \mathrm{~mm}$ to $5 \mathrm{~mm})$, the technique to restore the original tensile strength of the cracked element is by pressure injection of epoxy, briefly explain in retrofitting technique topic. For cracks wider than about $5 \mathrm{~mm}$ or for regions in which the concrete or masonry has crushed, a treatment other than injection is indicated is 
also briefly explained in the retrofitting technique topic. Cracks can be further classified as, flexure cracks, shear cracks, sliding, or combined. As narrated by Mukherjee Abhijit and Joshi Mangesh [4] fibre composites are effective for repair of minor and medium crack in R.C.C. structures.

\section{Post earthquake damage evaluations}

\subsection{Emergency earthquake damage evaluation}

Immediately after a damaging earthquake, an initial evaluation of each structure will be made by the official inspection team to determine quickly the general level of damage to the structure and if the structure is safe for continued occupancy.

Based on this initial evaluation each examined structure may be in one of the main categories labeled as follows:

i Green - This category is for buildings whose original seismic capacity has not been decreased and which do not appear to pose any danger to human life. The buildings are immediately usable and the entry unlimited. These building may have sustained slight damage requiring repair.

ii Yellow - Buildings in this category have decreased seismic capacity. Limited entry at owner's risk is permitted but not usage on a continuous basis. The need for supporting and protection of both the building and its surrounding should be considered.

iii Red - Buildings in this category are unsafe as subject to sudden collapse. Entry is prohibited and building surroundings should be protected. Decision for demolition will be made on the basis of a more thorough inspection after investigating technical possibilities for repair and for strengthening and their economic justification.

\subsection{Preliminary investigation}

The main purpose of the preliminary investigation of the state of the structure is to determine in detail the nature and degree of damage and to design and install emergency measures for temporary support to avert the risk of casualties and injuries, as well as to minimize the possible material losses in case of increased damage to the structure. The probability of repeated seismic activities during the days after the first shock is quite enough. Moreover, increased damage resulting from earthquake effects is often due to the dead and live loads where the continuity of the stress path of the forces to the foundations is partially or fully interrupted. The preliminary investigation will also be utilized in determining repair and /or strengthening measure.

\section{Case study of a residential building structure}

The building is located in South Gujarat region. It is designed for a ground level plus six floors, all under construction. Presently, ground plus three floors of the 
reinforced concrete building are constructed. The building was severly damaged during the $26^{\text {th }}$ January 2001 Bhuj earthquake (magnitude 7.2 Richter scale, duration 108 seconds). The building was approximately 400 kilometres away from the epicentre, but still large cracking and damage was observed. The building structure is analytically solved with different types of retrofitting methods. Figure 1 shows the typical plan of the building and all structural details of the building are enclosed in the envelope. As seen in plan, there are four flats on each floor and separated by stair portion. The expansion joint is provided between flat portion and stair portion.

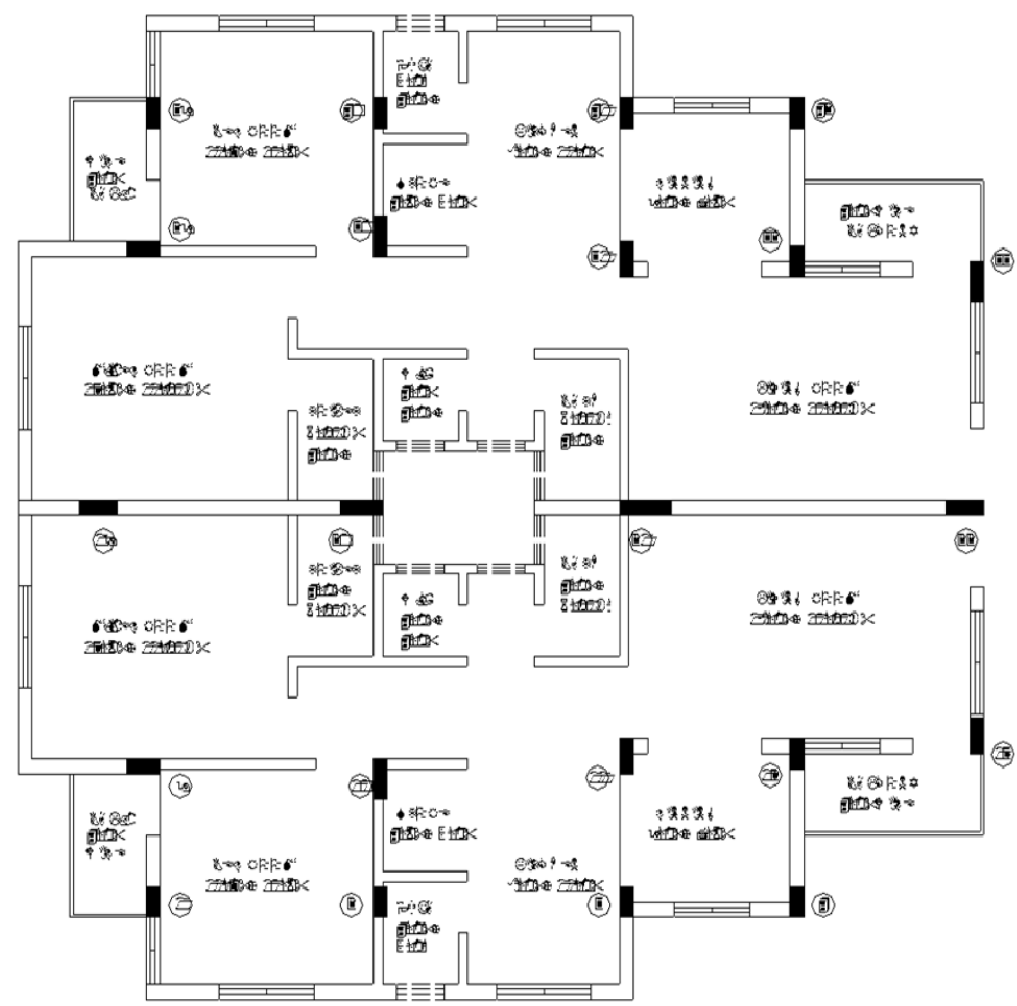

Figure 1: $\quad$ Plan of the building.

Three techniques are used for analysis to achieve a comprehensive strengthening of the structure as a whole. These techniques are

i Retrofitting by Reinforced concrete Jacketing.

ii Retrofitting by Adding Diagonal Bracings.

iii Retrofitting by new Shear walls.

The other techniques are not suitable for the local corporation by laws, organizer commercial purposes and also some of them are not feasible. The STAAD -III is 
use for analysis Purpose. STAAD-III is comprehensive and flexible general purpose structural software that address to all aspects of structural engineering. The codes used for analysis and design are IS 456:2000 and IS 1893:1984. The compressive strength of old structure was measured employing Non Destructive Testing using Rebound Hammer method.

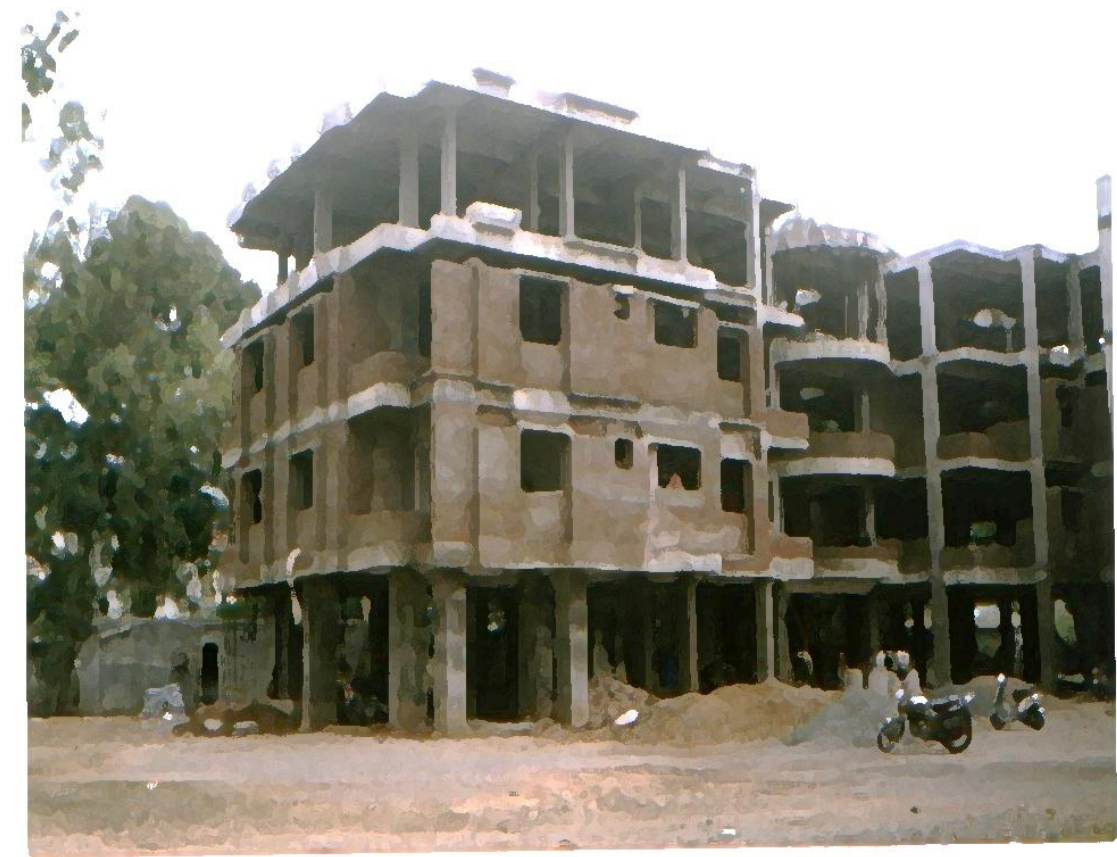

Figure 2: $\quad$ Photo of the building being constructed.

Different loading combinations for analysis and design are considered which basically comprises of dead loads, live loads and earthquake loads.

Steps for structural analysis of the building with reinforced concrete jacketing:

i Analyze the building for Dead Load and Live load with actual site member properties.

ii Analyze the building for Dead load, Live load and Lateral load in four directions with actual site member properties.

iii Analyze the building after applying Jacket to all columns (vertical member) at ground floor with Dead load, Live load and Lateral load.

iv Analyze the building after applying Jacket to all columns (vertical member) at Ground Floor and only corner columns at first floor with dead load, Live load and Lateral load. Analyze the building after applying Jacket to all columns (vertical member) at Ground Floor and only periphery columns at first floor_with Dead load, Live load and Lateral load. 

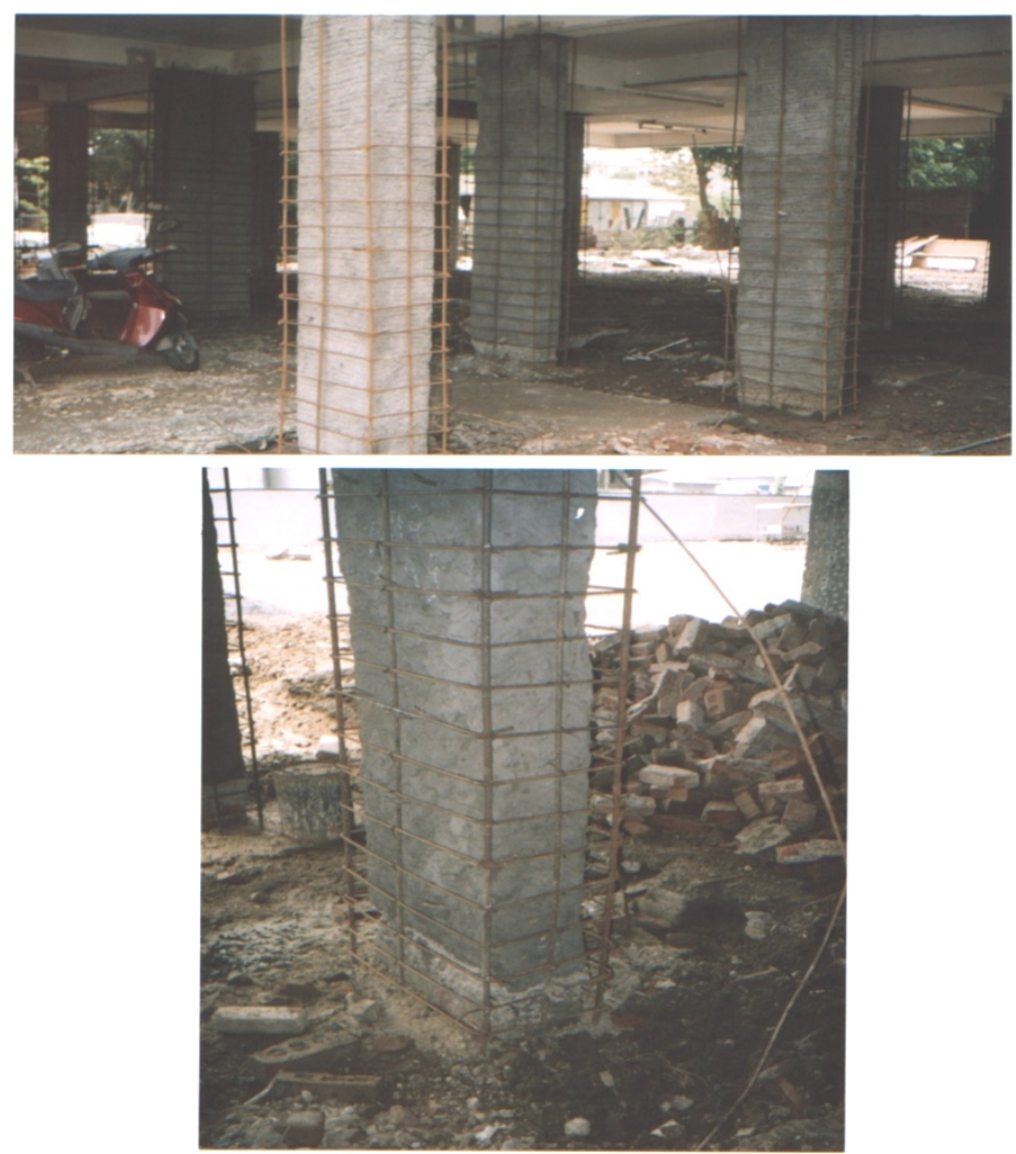

Figure 3: Jacketing of columns.

$\mathrm{V}$ Analyze the building after applying Jacket to all columns (vertical member) at First Floor with Dead load, Live load and Lateral load.

vi Analyze the building after applying Jacket to the columns (vertical member) which are biaxial fail at Second floor with Dead load, Live load and Lateral load.

vii Analyze the building after applying Jacket to all columns (vertical member) Second floor with Dead load, Live load and Lateral load.

viii Analyze the building after applying Jacket to all columns (vertical member) up to Second floor and only corner columns at Third floor with Dead load, Live load and Lateral load.

ix Analyze the building after applying Jacket to all columns (vertical member) up to Second floor and only periphery columns at Third floor with Dead load, Live load and Lateral load.

$\mathrm{x}$ Analyze the building after applying Jacket to all columns (vertical member) up to Third floor with Dead load, Live load and Lateral load. 
xi Analyze the building after applying Jacket to all columns (vertical member) up to Third floor and only periphery columns at Fourth floor with Dead load, Live load and Lateral load.

xii Analyze the building after applying Jacket to all columns (vertical member) up to First floor and only the columns which are biaxial fail up to Fourth floor with Dead load, Live load and Lateral load

\subsection{Application of reinforced concrete jacketing to the building structure}

Reinforced concrete jacketing according the available space conditions around the columns can be performed by adding jacketing to one, two, three or four sides of concrete column sections (Fig. 3). It is strongly recommended that columns be jacketed on all four sides for best performance in future earthquakes. In order to achieve the best bond between the new and the existing concrete, four sided jacketing is also most desirable. In case one, two, or three sided jacketing is all that is possible, the concrete cover in the jacketed parts of the existing column must be chipped away so new ties can be welded to existing ties.

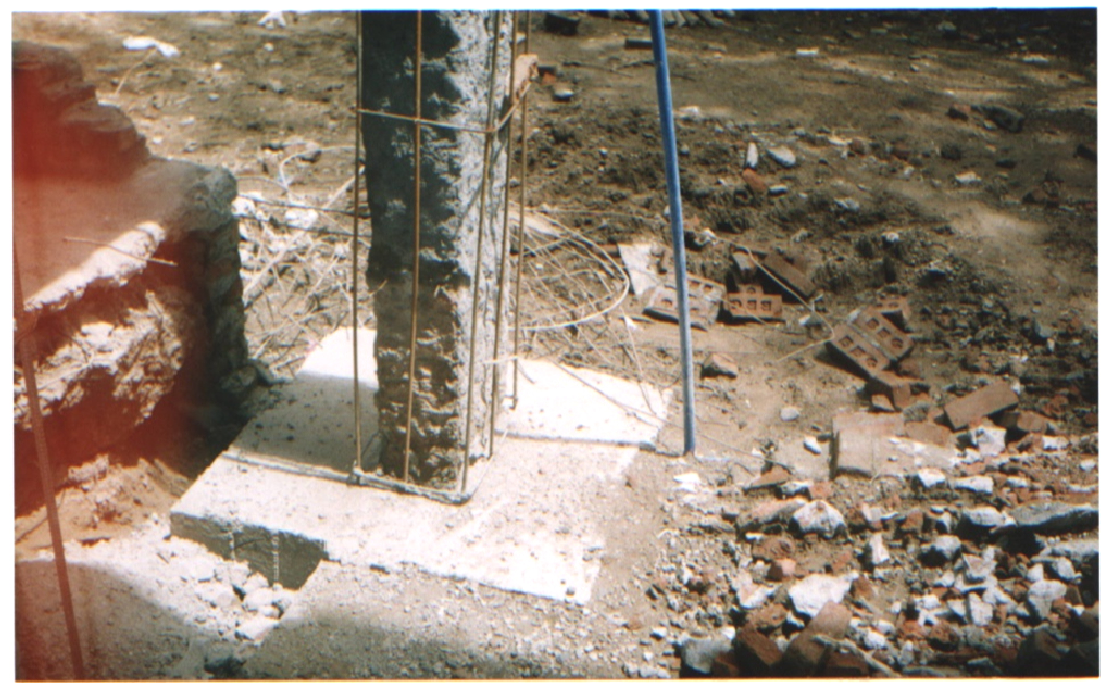

Figure 4: New pad foundations.

\subsection{Addition of diagonal braces in the building}

Diagonal bracing is used when there is a requirement of a moderate strength increase, while a great ductility and stiffness enhancement of the whole structure is desirable. Main advantages of the technique are speed of application, the uninterrupted natural lighting of the space. When brick-masonry walls already exist in the selected frame bays, the method can be applied by adding the bracing system externally on the frame or to demolish the existing masonry wall then provide bracing and then again fill with masonry wall. 


\subsection{Addition of shear walls in the building}

Cast-in-situ shear walls are constructed in properly selected frame bays of the structural system between the existing columns and beams of the frame. The walls are generally cast-in-situ but may be installed with shot-crete. Precast, prefabricated concrete elements can be used. The favorable position of new added element (Shear wall) should be in such a way that the centre of mass of the building and centre of stiffness of the building coincide or the distance between centre of mass of the building and centre of stiffness of the building be less. Even though bracing is provided up to the fourth floor, the columns which are perpendicular to the $\mathrm{x}$-axis fail in biaxial bending. So, it is advisable to add a jacketing system (i.e. combination of the system). Hence, the building can resist future earthquake loads.

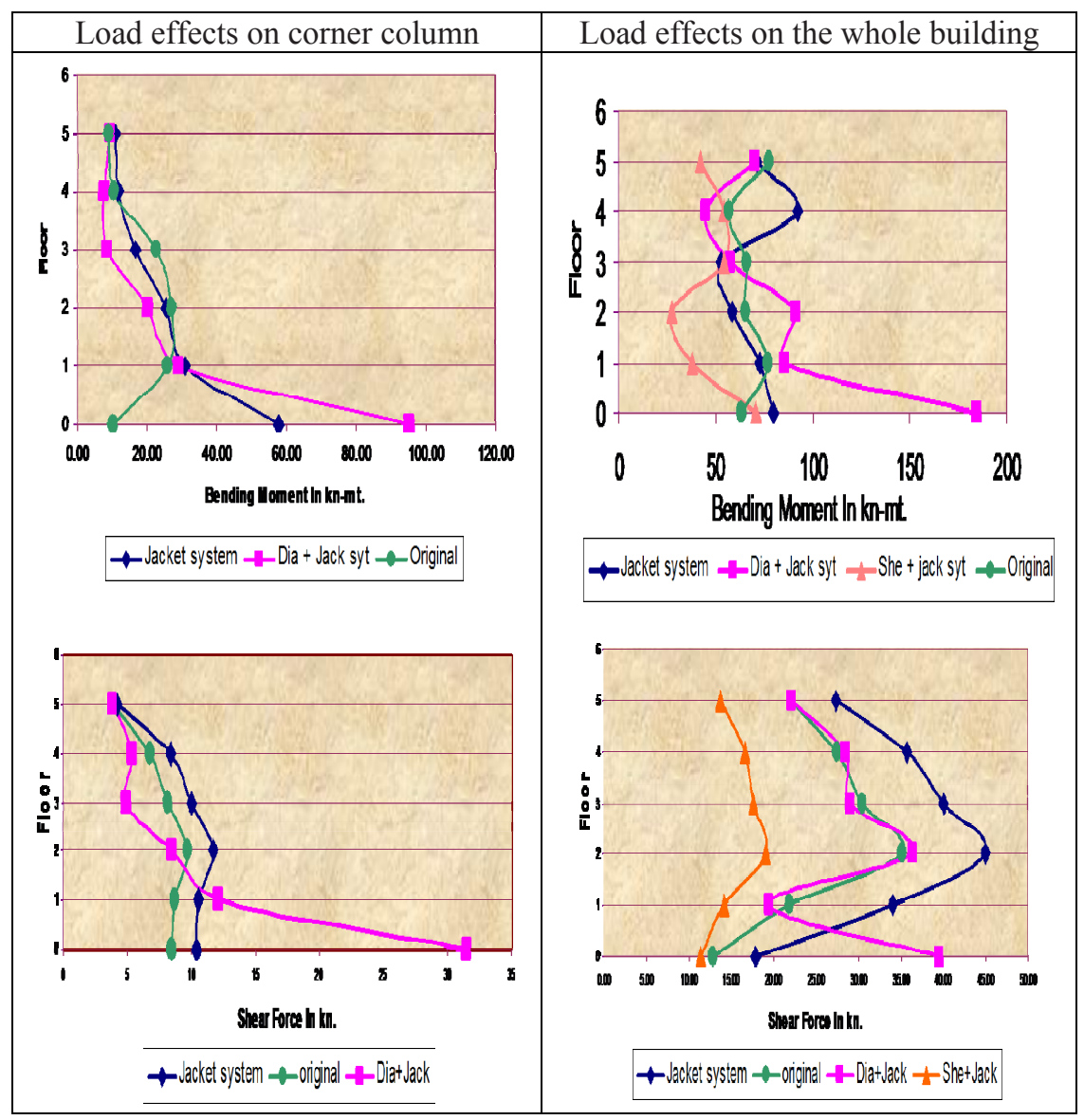

Figure 5: Results (load effects) showing comparison between the retrofitting methods used. 


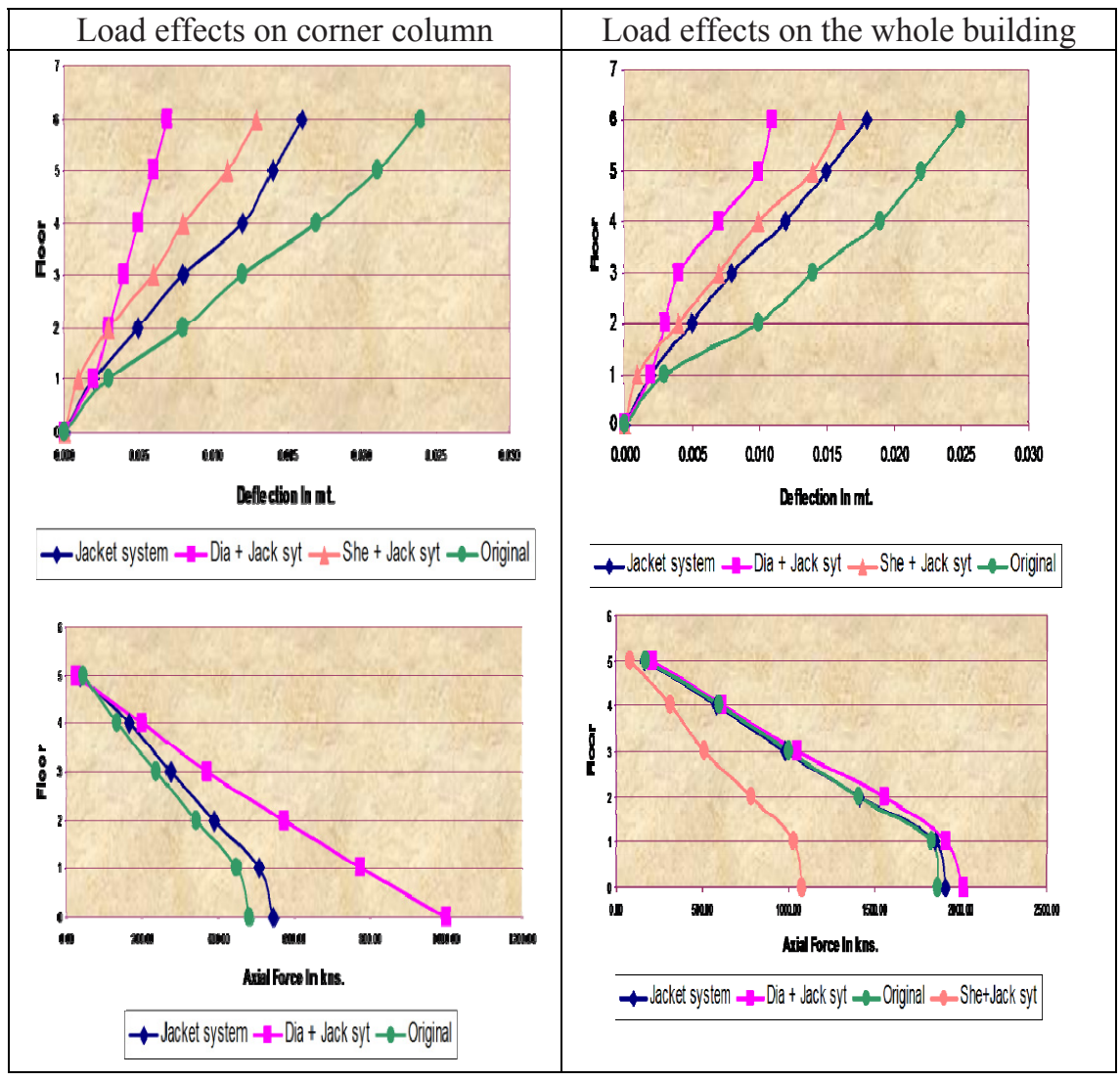

Figure 5: Continued.

\section{Results and discussion}

The results obtained from the output of Staad-III various graphs are plotted. The graphs are plotted floor level versus Bending Moment, Shear Force, Deflection, and Axial Forces of the building. On the X-axis Bending Moment (kN-mt), Shear Force $(\mathrm{kN})$, Deflection $(\mathrm{mt})$, and Axial Force $(\mathrm{kN})$ and on the Y-axis different floor levels of the building are plotted.

i The choice of a suitable retrofitting methods and appropriate strengthening technique is not an easy matter, ever since intervention is a unique case. This has a parallel saying in medicine, "There are no diseases but patients". Here, this can be restated as, "There are no structural deficiencies, but deficient structure".

ii Undoubtedly, for the above case study, the retrofitting methods aimed at achieving comprehensive strengthening of the structure as a whole gives better solution. 
iii As a thumb rule, if the cost of strengthening and retrofitting is less than about $50 \%$ of the reconstruction cost, the retrofitting is adapted. So, retrofitting methods for this building is recommended as it worked out so.

iv Addition of new element (shear wall) is a better solution for a particular building due to the following reason;

a. Bending moment is less in Shear wall plus Jacketing method.

b. Shear force is also less in Shear wall plus Jacketing system.

c. In deflection, the Shear wall plus Jacketing system is second then the diagonal bracing plus jacketing system.

d. As per cost analysis, the cost of Shear wall plus Jacketing methods is less.

e. The form work for the shear wall methods is also easy.

\section{References}

[1] Shah, Desai and Mehta, Handling post-earthquake challenges in Gujarat, India, Presentation documents of one day seminar at CEPT, June 2001.

[2] IS: 13935-1993, Indian Standard Code of Repair and Seismic strengthening of building guidelines, Bureau of Indian Standards, New Delhi.

[3] Jain Sudhir K. and Murty C.V.R., Seismic design and Retrofitting of reinforced concrete buildings, Short course at Ahmedabad by Continuing education programme, Indian institute of technology Kanpur, December 2001.

[4] Mukergee Abhijit and Joshi Mangesh V., Seismic retrofitting technique using fiber composites, The Indian Concrete Journal, Vol.- 76, No - 8, pp. 496-502, August 2002. 